\title{
Pegada de Carbono de propriedades agroecológicas do Agreste Meridional de Pernambuco
}

As atividades agrícolas têm acarretado em impactos ambientais, principalmente, por ser um dos setores que mais emitem Gases do Efeito Estufa (GEE). A agricultura baseada na prática e princípios da Agroecologia fortalece as relações com o Meio Ambiente e se apresenta como uma alternativa para reverter o aquecimento global e as mudanças climáticas. Diante disso, este estudo objetivou analisar o sequestro de carbono em agroecossistemas familiares. Foram avaliadas nove propriedades rurais familiares em transição agroecológica, localizadas na região do Agreste Meridional de Pernambuco. Os dados foram obtidos a partir de estudo de campo, como também, por análise de imagens do software Google Earth. Desse modo, verificou-se a dimensão das propriedades e cultivos agrícolas. Por sua vez, as árvores e arbustos foram quantificadas e também classificadas quanto ao porte. Também se levou em conta as informações sobre a periodicidade e quantidade de insumos. Foi calculado o valor estimado de sequestro de carbono anual em quilos de Dióxido de Carbono (CO2) por meio da plataforma digital Positive Climate Design. Posteriormente, foi calculado o valor da eficiência do sequestro de CO2. A partir dos resultados obtidos, observou-se que as propriedades apresentam capacidade relevante no sequestro de carbono. As atividades como o manejo do solo, a diversidade vegetal, rotação de culturas e o controle natural de pragas, doenças e ervas espontâneas colaboraram com a Pegada de Carbono nestas propriedades. Além disso, tais sistemas podem contribuir com as metas de redução de GEE firmadas pelo país, valorizando assim, a terra e seus produtos.

Palavras-chave: Aquecimento global; Mudanças climáticas; Sistemas agroecológicos.

\section{Carbon Footprint of agroecological properties of the Agreste Meridional of Pernambuco}

Agricultural activities have resulted in environmental impacts, mainly because it is one of the sectors that emit the most Greenhouse Gases (GHG). Agriculture based on the practice and principles of Agroecology strengthens relations with the Environment and presents itself as an alternative to reverse global warming and climate change. Therefore, this study aimed to analyze carbon sequestration in family agroecosystems. Nine family farms in agroecological transition, located in the Agreste Meridional region of Pernambuco, were evaluated. The data were obtained from a field study, as well as through image analysis of the Google Earth software. In this way, the size of agricultural properties and crops was verified. In turn, the trees and shrubs were quantified and also classified according to their size. Information on the frequency and quantity of inputs was also taken into account. The estimated annual carbon sequestration value in kilograms of carbon dioxide (CO2) was calculated using the Positive Climate Design digital platform. Subsequently, the efficiency of $\mathrm{CO} 2$ sequestration was calculated. From the results obtained, it was observed that the properties have a relevant capacity in carbon sequestration. Activities such as soil management, plant diversity, crop rotation and natural control of pests, diseases and spontaneous herbs collaborated with the Carbon Footprint in these properties. In addition, such systems can contribute to the GHG reduction targets set by the country, thus valuing the land and its products.

Keywords: Global warming; Climate changes; Agroecological systems.

Topic: Conservação da Biodiversidade

Reviewed anonymously in the process of blind peer.

Luciano Pires de Andrade (D)

Universidade Federal do Agreste de Pernambuco, Brasil http://lattes.cnpq.br/5367851306746294

http://orcid.org/0000-0001-5818-711X

lucianopandrade@gmail.com

Romário Nunes da Silva

Universidade Federal do Agreste de Pernambuco, Brasil

http://orcid.org/0000-0002-0401-8000

romario.nuness@gmail.com

Lucas de Lucena Rocha

Universidade Federal do Agreste de Pernambuco, Brasil http://lattes.cnpq.br/3728121678760017

http://orcid.org/0000-0001-7600-8293

lucasdelucenarocha@gmail.com
Received: 08/04/2021

Approved: 07/05/2021

\author{
Horasa Maria Lima da Silva Andrade (ID \\ Universidade Federal do Agreste de Pernambuco, Brasil \\ http://lattes.cnpq.br/4314101991387960 \\ http://orcid.org/0000-0002-5366-6610 \\ horasaa@gmail.com
}

Referencing this:

ANDRADE, L. P.; SILVA, R. N.; ROCHA, L. L.; ANDRADE, H. M. L. S. Pegada de Carbono de propriedades agroecológicas do Agreste Meridional de Pernambuco. Revista Ibero Americana de Ciências Ambientais, v.12, n.5, p.151-158, 2021. DOI: http://doi.org/10.6008/CBPC2179-6858.2021.005.0013 


\section{INTRODUÇÃO}

Atualmente as atividades agrícolas têm contribuído para a diminuição da biodiversidade. Manejos como o desmatamento da área e a aplicação de defensivos agrícolas podem atingir áreas vizinhas além de onde se encontra o cultivo, e com a predominância da monocultura, que empobrece o solo, a produtividade é diminuída com o tempo. De modo geral, o manejo inadequado e a mecanização acarretam na erosão e compactação do solo, diminuindo sua permeabilidade, e também contribuindo com o assoreamento de rios. O maquinário agrícola por sua vez, utiliza combustíveis fósseis, e a sua queima contribui para a emissão de Gases de Efeito Estufa (GEE) (MILLER et al., 2012).

A agricultura é também, uma das atividades que mais consomem água doce no mundo a partir da irrigação. O uso em excesso desse recurso pode acarretar em escassez hídrica, principalmente em regiões onde já existe uma baixa oferta de água (FAO, 2018). Já os fertilizantes utilizados no plantio podem impactar o Meio Ambiente de diversas formas. Os excessos de fertilizantes nitrogenados e fosfatados, carreados por lixiviação ou escoamento superficial, podem acarretar na eutrofização de corpos hídricos, o que afeta a qualidade da água. Em especial os fertilizantes nitrogenados são responsáveis pela emissão de GEE. A produção de fertilizantes e defensiva agrícola pode causar a emissão de metais pesados, o que contribui com o aumento da toxicidade do solo e da água (BAUMANN et al., 2004).

Dentre os GEE emitidos pelas atividades humanas, o gás carbônico $\left(\mathrm{CO}_{2}\right)$ é responsável por aproximadamente $70 \%$ do potencial de elevação da temperatura terrestre. Apenas nos últimos 250 anos, o aumento da concentração de $\mathrm{CO}_{2}$ na atmosfera foi de $31 \%$, chegando aos atuais 366 partes por milhão (ppm), nível mais alto observado nos últimos 420 mil anos. No último século, a temperatura do planeta já subiu 0,70 C e, para os próximos cem anos, a projeção do IPCC - Intergovernamental Panel on Climate Change é que possa haver um aumento entre 1,4으 C 5,8 C (MARENGO, 2006).

Muitos estudos voltados à identificação de fontes e sumidouros de GEE têm sido aplicados em amplas escalas espaciais, buscando entender os perfis das emissões pautadas geralmente em produtos específicos, indivíduos, cidades ou países (ROBINSON et al., 2018). Recentemente diversos instrumentos foram desenvolvidos no intuito de descrever e quantificar tanto os efeitos diretos, como os indiretos da apropriação humana sobre o meio ambiente, bem como, os impactos decorrentes destes. Dentre esses instrumentos destaca-se a Pegada de Carbono (PATTERSON et al., 2017). Segundo Høgevold (2011), a Pegada de Carbono trata-se de um indicador que é frequentemente relatado em estudos de avaliação do ciclo de vida. Sua aplicação também está diretamente ligada à quantificação da liberação de $\mathrm{CO}_{2}$ ou GEE emitidos de um produto ou processo, colaborando com a reflexão em torno das potenciais influências do aquecimento global ou mudança climática (KAVEHEl et al., 2018).

No Brasil, os estudos voltados à Pegada de Carbono são bem pontuais e há diversas lacunas a serem compreendidas. Entre estas, a quantificação dos impactos dos cultivos realizados em agroecossistemas familiares que estão em transição agroecológica. Enquanto estruturas de mercados, o ambiente da agricultura familiar se destaca ao optar por uma produção baseada na prática e princípios da Agroecologia, o que enaltece a diversificação produtiva e fortalece as relações com o Meio Ambiente. 
Assim, essa escolha vai de encontro aos modelos exógenos da modernização agrícola (NUNES et al., 2018).

Desse modo, o presente trabalho tem como objetivo analisar o sequestro de carbono em agroecossistemas localizados na região do Agreste Meridional de Pernambuco, buscando contribuir com o conhecimento a respeito do sequestro de carbono pelas atividades agrícolas da agricultura familiar, que hoje apresenta resistência no uso de manejo que causa a degradação ambiental.

\section{METODOLOGIA}

\section{Área de estudo}

Durante os meses de fevereiro e março de 2021 foram avaliadas nove propriedades rurais familiares que estão em transição agroecológica, localizadas nos seguintes municípios: Bom Conselho, Jucati e Saloá. Esses municípios estão situados na região Agreste Meridional de Pernambuco (Figura 1), que ao todo compreende 28 municípios e possui uma extensão territorial de $13 \mathrm{mil} \mathrm{Km}^{2}$. A maior parte de sua área varia entre 400 e $800 \mathrm{~m}$, chegando até $1.100 \mathrm{~m}$, os chamados brejos de altitude e a sua vegetação é densa, sendo contemplada pela caatinga hiperxerófila, contando ainda com a presença de espécies decíduas e de cactáceas e bromélias. O principal uso do solo se dá pela agricultura familiar de subsistência. Também é conhecida como a bacia leiteira de Pernambuco, por contar com a maior produção de leite do Estado (SILVA et al., 2011; TORRES et al., 2014; BRASIL, 2015; SILVA et al., 2016).

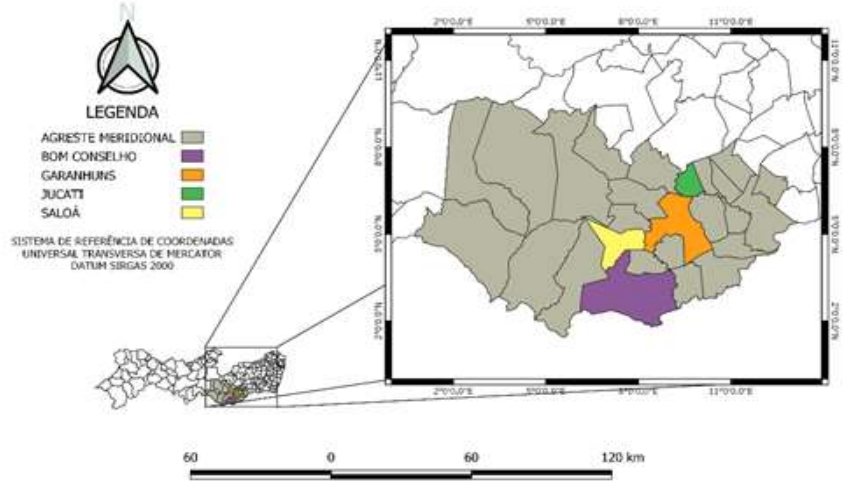

Figura 1: Localização dos municípios pertencentes à região Agreste Meridional de Pernambuco.

Todas as propriedades se encontram em transição agroecológica. Os agricultores participam de um projeto de pesquisa e extensão denominado Agrofeira- Feira Territorial da Agroecologia e Agricultura Familiar, para comercialização dos produtos, que é realizada pelo Núcleo Agrofamiliar, da Universidade Federal Rural de Pernambuco/Universidade Federal do Agreste de Pernambuco (UFAPE) com diversas instituições públicas e entidades da sociedade civil. A feira acontece há mais de cinco anos na cidade de Garanhuns/Pernambuco e, por meio desta parceria, os agricultores são acompanhados e capacitados por professores e alunos da universidade.

No âmbito de preservar a identidade dos proprietários e de facilitar o tratamento dos dados e a organização dos resultados, as propriedades foram denominadas da seguinte forma: Agroecossistema 1 (AG1), Agroecossistema 2 (AG2), Agroecossistema 3 (AG3), Agroecossistema 4 (AG4), Agroecossistema 5 
(AG5), Agroecossistema 6 (AG6) no município de Garanhuns; Agroecossistema 7 (AG7) - no município de Saloá; Agroecossistema 8 (AG8) no município de Bom Conselho; Agroecossistema 9 (AG9) no município de Jucati.

\section{Procedimentos metodológicos}

A coleta das informações se deu a partir de um estudo em campo nas nove propriedades agrícolas como também por imagens dos satélites Landsat 5 e Landsat 8, através do software Google Earth. Desse modo verificou-se a dimensão das propriedades e cultivos agrícolas. Por sua vez, as árvores e arbustos foram quantificadas e também classificadas quanto ao porte, se são perenifólias ou caducifólias. Também se levou em conta as informações sobre periodicidade e quantidade de insumos como fertilizantes e defensivos.

Após a obtenção de todos os dados, foi calculado o valor estimado de sequestro de carbono anual em quilos $(\mathrm{Kg})$ de $\mathrm{CO}_{2}$ de acordo com os dados numéricos fornecidos pela plataforma digital Positive Climate Design, para o Hemisfério Sul do Globo Terrestre. E posteriormente calculou-se também o valor da eficiência do sequestro de $\mathrm{CO}_{2}$ de cada agroecossistema através da divisão do valor do módulo do sequestro de carbono pelo valor da extensão da propriedade em metros quadrados.

Quadro 1: Valores de sequestro de $\mathrm{CO}_{2}$ anual por $\mathrm{m}^{2}$ de pastagem - valores para a Zona Sul do Globo Terrestre.

\begin{tabular}{|l|l|l|}
\hline Sem manutenção & Manutenção moderada & Manutenção intensiva \\
\hline$-40 \mathrm{Kg}$ de $\mathrm{CO}_{2} / \mathrm{ano}$ & $+2 \mathrm{Kg} \mathrm{de} \mathrm{CO}_{2} / \mathrm{ano}$ & $+10 \mathrm{Kg} \mathrm{de} \mathrm{CO}_{2} / \mathrm{ano}$ \\
\hline
\end{tabular}

Fonte: PATHFINDER (2021).

Quadro 2: Valores de sequestro de carbono por unidade de arbusto em $\mathrm{Kg}$ de $\mathrm{CO}_{2}$ por ano - valores para a Zona Sul do Globo Terrestre.

\begin{tabular}{|l|l|l|l|l|l|}
\hline Caducifólia pequena & Caducifólia média & Caducifólia grande & Perene pequena & Perene média & Perene grande \\
\hline$-50 \mathrm{Kg}$ de $\mathrm{CO}_{2} / \mathrm{ano}$ & $-102 \mathrm{Kg}$ de $\mathrm{CO}_{2} / \mathrm{ano}$ & $-202 \mathrm{Kg}$ de $\mathrm{CO}_{2} / \mathrm{ano}$ & $-13 \mathrm{Kg} \mathrm{de} \mathrm{CO} / \mathrm{ano}$ & $-26 \mathrm{Kg} \mathrm{de} \mathrm{CO}_{2} / \mathrm{ano}$ & $-2 \mathrm{Kg} \mathrm{de} \mathrm{CO}_{2} / \mathrm{ano}$ \\
\hline
\end{tabular}

Fonte: PATHFINDER (2021).

Quadro 3: Valores de sequestro de carbono por unidade de árvore em $\mathrm{Kg}$ de $\mathrm{CO}_{2}$ por ano - valores para a Zona Sul do Globo Terrestre.

\begin{tabular}{|c|c|c|c|c|c|}
\hline $\begin{array}{l}\text { Caducifólia } \\
\text { pequena }\end{array}$ & Caducifólia média & Caducifólia grande & Perene pequena & Perene média & Perene grande \\
\hline$-605 \mathrm{Kg}$ de $\mathrm{CO}_{2} / \mathrm{ano}$ & $\begin{array}{l}-2551 \mathrm{Kg} \text { de } \\
\mathrm{CO}_{2} / \mathrm{ano}\end{array}$ & $\begin{array}{l}-5623 \mathrm{Kg} \text { de } \\
\mathrm{CO}_{2} / \mathrm{ano}\end{array}$ & $\begin{array}{l}-93 \mathrm{Kg} \text { de } \\
\mathrm{CO}_{2} / \text { ano }\end{array}$ & $\begin{array}{l}-1861 \mathrm{Kg} \text { de } \mathrm{CO}_{2} \\
\text { /ano }\end{array}$ & $\begin{array}{l}-5693 \mathrm{Kg} \text { de } \\
\mathrm{CO}_{2} / \mathrm{ano}\end{array}$ \\
\hline
\end{tabular}

Fonte: PATHFINDER (2021).

\section{RESULTADOS E DISCUSSÃO}

A partir dos resultados obtidos verificou-se que a capacidade de sequestro de carbono pelas propriedades foi da seguinte maneira: AG1 (-654901); AG2 (-2.942575); AG3 (-1221538); AG4 (-639858); AG5 (-2925137); AG6 (-445438); AG7 (-637880); AG8 (-2911570); e AG9 (-234822) (Tabela 1). Vale ressaltar que estes valores não determinam a eficiência de sequestro de carbono pelos agroecossistemas quando se 
é considerado a extensão territorial dos mesmos em hectares (ha).

Tabela 1: Quantidade de sequestro de $\mathrm{CO}_{2}$ e extensão das propriedades.

\begin{tabular}{ccc}
\hline Agroecossistema & Sequestro de $\mathrm{CO}_{\mathbf{2}}$ (kg/ano) & Extensão Territorial (ha) \\
\hline AG1 & -654901 & 1,5 \\
\hline AG2 & -2942575 & 7 \\
\hline AG3 & -1221538 & 2,5 \\
\hline AG4 & -639858 & 1,5 \\
\hline AG6 & -2925137 & 7 \\
\hline AG7 & -445438 & 1 \\
\hline AG8 & -637880 & 1,5 \\
\hline AG9 & -2911570 & 7 \\
\hline
\end{tabular}

Obs. Elaborado a partir dos dados da pesquisa.

Conforme os dados apresentados na tabela 2 pode-se observar a eficiência das propriedades agroecológicas através da divisão do valor do módulo do sequestro de carbono pelo valor da extensão da propriedade em metros quadrados. A propriedade com 2,5 hectares (AG3) apresentou a maior capacidade de sequestro de carbono do estudo, com valor de $48,86 \mathrm{Kg}$ de $\mathrm{CO}_{2} / \mathrm{m}^{2}$. A menor propriedade (AG9), com 0,5 hectare, apresentou o segundo melhor desempenho em sequestro de carbono do estudo, com 46,96 $\mathrm{Kg}$ de $\mathrm{CO}_{2} / \mathrm{m}^{2}$. A segunda menor propriedade da análise com 1 hectare (AG6) apresentou o terceiro melhor desempenho em sequestro de carbono do estudo, com índice de $44,54 \mathrm{Kg}$ de $\mathrm{CO} 2 / \mathrm{m} 2$. As propriedades de 1,5 hectare apresentaram índices de 43,66 Kg de $\mathrm{CO}_{2} / \mathrm{m}^{2}$ (AG1), 42,65 $\mathrm{Kg}$ de $\mathrm{CO}_{2} / \mathrm{m}^{2}$ (AG4) e 42,52 $\mathrm{Kg}$ de $\mathrm{CO}_{2} / \mathrm{m}^{2}$ (AG7). $\mathrm{E}$ as maiores propriedades, com 7 hectares de extensão (AG2, AG5 e AG8), obtiveram os índices mais baixos de eficiência em sequestro de carbono do estudo com 41,77 Kg de CO $2 / \mathrm{m}^{2} ; 41,78 \mathrm{Kg}$ de $\mathrm{CO}_{2} / \mathrm{m}^{2} ; 41,59 \mathrm{Kg}$ de $\mathrm{CO}_{2} / \mathrm{m}^{2}$, respectivamente.

Tabela 2: Desempenho de sequestro de $\mathrm{CO}_{2}$ e extensão da propriedade.

\begin{tabular}{ccc}
\hline Desempenho de sequestro de $\mathbf{C O}_{2} / \mathbf{m}^{2}$ & Extensão da propriedade (ha) & Agroecossistema \\
\hline $48,86 \mathrm{Kg}$ & 2,5 & AG3 \\
\hline $46,96 \mathrm{Kg}$ & 0,5 & AG9 \\
\hline $44,54 \mathrm{Kg}$ & 1 & AG6 \\
\hline $43,66 \mathrm{Kg}$ & 1,5 & AG1 \\
\hline $42,65 \mathrm{Kg}$ & 1,5 & AG4 \\
\hline $42,52 \mathrm{Kg}$ & 1,5 & AG7 \\
\hline $41,78 \mathrm{Kg}$ & 7 & AG5 \\
\hline $41,77 \mathrm{Kg}$ & 7 & AG2 \\
\hline $41,59 \mathrm{Kg}$ & 7 & AG8 \\
\hline
\end{tabular}

Obs. Elaborada a partir dos dados da pesquisa.

Esses resultados demonstram que a capacidade de sequestro de carbono das propriedades que são objetos empíricos deste estudo, é inversamente proporcional à extensão territorial da propriedade, com exceção da propriedade AG3, com melhor desempenho em sequestro de carbono. A presença de várias árvores nos sistemas produtivos das propriedades, em particular as menores, colaborou com o bom resultado do sequestro de carbono. Sabe-se que, os sistemas agrícolas nos quais são reintroduzidas espécies arbóreas manejadas juntas com outras culturas e, ou, animais possuem alto potencial de absorver $\mathrm{CO}_{2}$ (ALBRECHT et al., 2003).

Se tratando das atividades agrícolas realizadas nos agroecossistemas, realizam-se práticas como 
adubação orgânica (composto de esterco bovino e adubação verde), revolvimento mínimo do solo e rotação/sucessão de culturas. As etapas de preparo do solo, plantio e colheita são realizadas manualmente, não existindo o uso de máquinas de tração mecânica/elétrica. Tais práticas são benéficas e podem resultar em aumento nos estoques de carbono no solo, na vegetação, como também, podem reduzir a emissão de GEE na atmosfera (CARVALHO et al., 2010). O acúmulo de matéria orgânica nos solos de sistemas agrícolas e, consequentemente, o seu potencial para a remoção de $\mathrm{CO}_{2}$, já foi indicado em vários estudos realizados em diferentes regiões brasileiras (VILELA et al., 2011; ASSAD et al., 2013; PINTO et al., 2014).

O solo é um compartimento chave no processo de emissão e sequestro de carbono, uma vez que, há duas a três vezes mais carbono nos solos em relação ao estocado na vegetação e duas vezes mais em comparação à atmosfera. Portanto, manejos inadequados podem acarretar em desastres como a mineralização da matéria orgânica e emissão em grandes quantidades de GEE para a atmosfera. Isto demonstra o grau de importância que o bom manejo dos solos representa para o planeta atualmente (CERRI et al., 2007).

Os cultivos das propriedades são bastantes diversos. Destacam-se a produção de várias hortaliças como: coentro (Coriandrum sativum L.) cebolinha (Allium schoenoprasum L.) alface (Lactuca sativa L.), couve (Brassica oleracea L.) e cenoura (Daucus carota L.). Cultivam-se também algumas culturas anuais como: feijão (Phaseolus vulgaris L.) e milho (Zea mays ssp.) e também há produção de frutas como: goiaba (Psidium guajava L.), acerola (Malpighia emarginata DC) e manga (Mangifera indica L.). Durante o manejo dessas culturas, as necessidades por insumos externos são baixas, especialmente os fertilizantes e produtos químicos, os quais são substituídos por compostos orgânicos e defensivos naturais. Quando comparado ao manejo convencional, essas práticas além de apresentarem poucos impactos negativos ao Meio Ambiente colaboram com o sequestro de carbono e contribui na mitigação das emissões de gases na atmosfera (MARASENI et al., 2007).

A atividade da irrigação nas propriedades acontece de duas maneiras: manual e/ou gotejamento. Neste último caso, a água é distribuída apenas pela força da gravidade, o que não exige o uso de bomba elétrica ou a diesel. Lal (2005) ressalta que as práticas agrícolas que envolvem o uso de combustível fóssil, energia elétrica, aragem, fertilizantes e pesticidas, contribuem substancialmente para emissão de GEE. A conversão do arado para o plantio direto, o manejo integrado de pragas e o uso da água por meio de irrigação por gotejamento e práticas de subirrigação pode diminuir a emissão desses gases.

Os resultados apresentados neste estudo contribuem com a compreensão da dinâmica do sequestro de carbono em propriedades de sistema de produção agroecológico. Alguns trabalhos desenvolvidos em vários lugares do mundo já têm demonstrado a potencialidade que sistemas de produção agrícola alternativos ao convencional possuem com a Pegada de Carbono. Um exemplo foi o estudo comparativo do sequestro de carbono no sistema de produção orgânica e convencional de arroz na Indonésia, onde Komatsuzaki e Syuaib (2010) observaram que o solo do sistema de cultivo orgânico apresentou maior teor de carbono em relação aos solos convencionais. Esse resultado foi parecido com o que foi obtido por Shirato et al. (2005) em arrozais na Tailândia. Portanto, essas "agriculturas alternativas" 
podem ajudar não somente na mitigação do aquecimento global, mas também, estabelecer um sistema alimentar sustentável (KOMATSUZAKI et al., 2010).

\section{CONCLUSÕES}

Este estudo demonstrou que além de movimentar a dinâmica econômica territorial, os sistemas de produção agroecológica do Agreste Meridional de Pernambuco são aliadas para diminuição do aquecimento global e mudanças climáticas ao possuírem boas condições de realizar o sequestro de carbono. Porém, quando se levou em consideração o tamanho dos agroecossistemas, na maioria das vezes verificou-se uma desproporcionalidade, em que propriedades menores possuem maior eficiência de Pegada de Carbono.

Apesar de ainda ser um grande emissor de GEE no Brasil, o setor da agricultura apresenta um relevante potencial de redução em vários subsetores e processos. Nas atividades de propriedades agroecológicas, por exemplo, os sistemas integrados e as estratégias de manejo do solo, a diversidade vegetal, rotação de culturas e o controle natural de pragas, doenças e ervas espontâneas se apresentam como meios de redução de emissões e sequestro de GEE. Além disso, tais sistemas podem colaborar com as metas de redução de emissões desses gases firmadas pelo país, valorizando assim, a terra e seus produtos. A agricultura de base agroecológica pode ser considerada como uma eficaz mitigadora de GEE, além de aumentar a capacidade da produção. E por fim, tendo em vista a dinâmica dos sistemas produtivos, ressalta-se a necessidade de monitoramento do sequestro de carbono nesses agroecossistemas com o intuito de aperfeiçoar o processo de compreensão do sistema.

\section{REFERÊNCIAS}

ALBRECHT, A.; KANDJI, S. T.. Carbon sequestration in tropical agroforestry systems. Agriculture, ecosystems \& environment, v.99, n.1-3, p.15-27, 2003. DOI: http://doi.org/10.1016/S0167-8809(03)00138-5

ASSAD, E. D.; PINTO, H. S.; MARTINS, S. C.; GROPPO, J. D.; SALGADO, P. R.; EVANGELISTA, B.; VASCONCELLOS, E.; SANO, E. E.; PAVÃO, E.; LUNA, R.; CAMARGO, P. B.; MARTINELLI, L. A.. Changes in soil carbon stocks in Brazil due to land use: paired site comparisons and a regional pasture soil survey. Biogeosciences, v.10, n.10, p.6141-6160, 2013. DOI: http://doi.org/10.5194/bg-10-6141-2013

BAUMANN, H.; TILLMAN, A. M.. The hitch hiker's guide to LCA: an orientation in life cycle assessment methodology and application. Lund: Studentlitteratur, 2004.

BRASIL. Caderno Territorial 002 - Agreste Meridional de Pernambuco. Ministério do Desenvolvimento Agrário, 2015.

CARVALHO, J. L. N.; AVANZI, J. C.; SILVA, M. L. N.; MELLO, C. R. D. CERRI, C. E. P.. Potencial de sequestro de carbono em diferentes biomas do Brasil. Revista Brasileira de Ciência do Solo, v.34, n.2, p.277-290, 2010. DOI:

http://doi.org/10.1590/S0100-06832010000200001

CERRI, C. C.; CERRI, C. E. P.. Agricultura e Aquecimento global. Boletim Informativo da Sociedade Brasileira de
Ciência do Solo, v.32, n.1, p.40-44, 2007.

FAO. Food And Agriculture Organization 2050: a escassez de água em várias partes do mundo ameaça a segurança alimentar e os meios de subsistência, 2018.

HØGEVOLD, N. M.. A corporate effort towards a sustainable business model: a case study from the Norwegian furniture industry. European Business Review, v.23, n.4, p.392-400, 2011. DOI: http://doi.org/10.1108/09555341111145771

KAVEHEI, E.; JENKINS, G. A.; ADAME, M. F.; LEMCKERT, C.. Carbon sequestration potential for mitigating the carbon footprint of green stormwater infrastructure. Renewable and Sustainable Energy Reviews, v.94, p.1179-1191, 2018. DOI: http://doi.org/10.1016/j.rser.2018.07.002

KOMATSUZAKI, M.; SYUAIB, M. F.. Comparison of the farming system and carbon sequestration between conventional and organic rice production in West Java, Indonesia. Sustainability, v.2, n.3, p.833-843, 2010. DOI: http://doi.org/10.3390/su2030833

LAL, R.. Carbon emission from farm operations. Environment international, v.30, n.7, p.981-990, 2004. DOI: http://doi.org/10.1016/j.envint.2004.03.005

MARASENI, T. N.; COCKFIELD, G.; APAN, A. A.. comparison of 
greenhouse gas emissions from inputs into farm enterprises in Southeast Queensland, Australia. Journal of

Environmental Science and Health, v.42, n.1, p.11-18, 2007. DOI: http://doi.org/10.1080/10934520601015354

Marengo, J. A.. Mudanças climáticas globais e seus efeitos sobre a biodiversidade: caracterização do clima atual e definição das alterações climáticas para o território brasileiro ao longo do século XXI. Brasília: MMA, 2006.

MILLER, G. T.; SPOOLMAN, S. E.. Ecologia e sustentabilidade. São Paulo: Cengage Learning, 2012.

NUNES, E. M. FRANÇA, A. R. M.; LIMA, J. S. S.; MEDEIROS, L. $S$. Novidades (Novelty) na Agricultura Familiar e sua associação com a agroecologia na produção de hortifrutigranjeiros no Território Sertão do Apodi (RN). Revista do Desenvolvimento Regional, v.23, n.1, p.213-236, 2018. DOI: http://doi.org/10.17058/redes.v23i1.9292

PATHFINDER. Climate Positive Design. Measure + Improve Our Carbon Impact, 2021.

PATTERSON, M.; MCDONALD, G.; HARDY, D.. Is there more in common than we think? Convergence of ecological footprinting, emergy analysis, life cycle assessment and other methods of environmental accounting. Ecological Modelling, v.362, p.19-36, 2017. DOI:

\section{http://doi.org/10.1016/jecolmodel.2017.07.022}

PINTO, H. S.; ASSAD, E. D.. Sumário executivo: mitigando mudanças climáticas no setor agrícola: estoque de carbono nos solos da Amazônia-Brasil. Brasília: Embrapa, 2014.
SHIRATO, Y.; PAISANCHAROEN, K.; SANGTONG, P.; NAKVIRO, C.; YOKOZAWA, M.; MATSUMOTO, N.. Testing the Rothamsted carbon model against data from long-term experiments on upland soils in Thailand. European Journal of Soil Science, v.56, n.2, p.179-188, 2005. DOI: http://doi.org/10.1111/j.1365-2389.2004.00659.x

SILVA, A. O.; MOURA, G. B. A.; SILVA, E. F. F.; LOPES, P. M. O.; SILVA, A. P. N.. Análise espaço-temporal da evapotranspiração de referência sob diferentes regimes de precipitações em Pernambuco. Revista Caatinga, v.24, n.2, p.135-142, 2011.

SILVA, Y. J. A. B. D.; NASCIMENTO, C. W. A. D.; SILVA, Y. J. A. B. D.; BIONDI, C. M.; SILVA, C. M. C. A.C.. Rare earth element concentrations in Brazilian Benchmark Soils. Revista Brasileira de Ciência do Solo, v.40, p.1-13, 2016. DOI: http://doi.org/10.1590/18069657rbcs20150413

TORRES, F. S. M.; PFALTZGRAFF, P. A. S.. Geodiversidade do estado de Pernambuco. Recife: CPRM, 2014.

VILELA, L.; MARTHA JUNIOR, G. B.; MACEDO, M. C. M.; MARCHAO, R. L.; GUIMARAES JUNIOR, R.; PULROLNIK, K.; MACIEL, G. A.. Sistemas de integração lavoura-pecuária na região do Cerrado. Pesquisa Agropecuária Brasileira, v.46, n. 10, p.1127-1138, 2011. DOI: http://doi.org/10.1590/S0100204X2011001000003

A CBPC - Companhia Brasileira de Produção Científica (CNPJ: 11.221.422/0001-03) detém os direitos materiais desta publicação. Os direitos referem-se à publicação do trabalho em qualquer parte do mundo, incluindo os direitos às renovações, expansões e disseminações da contribuição, bem como outros direitos subsidiários. Todos os trabalhos publicados eletronicamente poderão posteriormente ser publicados em coletâneas impressas sob coordenação da Sustenere Publishing, da Companhia Brasileira de Produção Científica e seus parceiros autorizados. Os (as) autores (as) preservam os direitos autorais, mas não têm permissão para a publicação da contribuição em outro meio, impresso ou digital, em português ou em tradução. 